\title{
Identification of macrophage inhibitory cytokine-1 (MIC-1) in adipose tissue and its secretion as an adipokine by human adipocytes
}

\author{
Q. Ding, T. Mracek, P. Gonzalez-Muniesa, K. Kos, J. Wilding, P. Trayhurn and C. Bing \\ Obesity Biology Research Unit, School of Clinical Sciences, University of Liverpool, Liverpool L69 3GA, UK
}

MIC-1, a divergent member of the transforming growth factor- $\beta$ superfamily ${ }^{(1)}$, is involved in the control of multiple cellular functions. Recently, MIC-1 has been implicated as a cachexia mediator inducing weight loss through the inhibition of appetite ${ }^{(2)}$. Adipose tissue as an endocrine organ secretes proteins (adipokines), which modulate appetite, nutrient metabolism, insulin sensitivity, stress responses and inflammation ${ }^{(3)}$. Given the diverse roles of MIC-1, adipose tissue could be important in the physiological function of this factor. The aim of the present study was therefore to examine whether MIC-1 is expressed in adipose tissue and whether it is a secretory product of adipocytes. The study also investigated factors that modulate MIC-1 production and the potential role of MIC- 1 in adipocytes.

Mouse adipose tissues were collected from different depots of C57B1/6 mice. Adipose tissue (visceral and subcutaneous) was also collected from human subjects with a wide range of BMI, undergoing general or bariatric surgery. For in vitro studies 3T3-L1 preadipocytes and human preadipocytes (Zen-Bio) were used and induced to differentiate into adipocytes in cell culture.

MIC-1 mRNA was detected in the major mouse adipose tissue depots (epididymal, perirenal, subcutaneous). In these depots MIC-1 gene expression was evident in both isolated mature adipocytes and stromal vascular cells. In 3T3-L1 adipocytes MIC-1 mRNA was detected before and after differentiation. Administration of leptin and IL-1 $\beta$ led to a reduction in MIC-1 mRNA levels (both $P<0.01$ ). Treatment with $\mathrm{H}_{2} \mathrm{O}_{2}$ induced a dose-dependent increase in MIC-1 mRNA $(P<0.01)$ while 15 -deoxy-12,14-PGJ $\left.{ }_{2}(15 \mathrm{~d}-\mathrm{PGJ})_{2}\right)$ caused a marked up-regulation of MIC-1 transcripts (19-fold; $P<0.001$ ).

MIC-1 mRNA and protein secretion were evident in human preadipocytes as well as differentiated adipocytes. MIC-1 gene expression and protein secretion by human adipocytes were stimulated by $\mathrm{H}_{2} \mathrm{O}_{2}$ and $15 \mathrm{~d}-\mathrm{PGJ}_{2}$ (all $P<0.01$ ). In addition, recombinant $\mathrm{MIC}-1$ increased adiponectin secretion by differentiated human adipocytes. MIC-1 mRNA and protein were also observed in human subcutaneous and visceral fat. MIC-1 mRNA levels were positively correlated with adiponectin mRNA in both visceral $(r 0.43, P<0.05, n 23)$ and subcutaneous $(r 0.65, P<0.01, n 16)$ depots. Moreover, MIC-1 mRNA was negatively associated with BMI $(r-0.53, P<0.01$, visceral; $r-0.56, P<0.05$, subcutaneous) and body fat mass $(r-0.47, P<0.05$, visceral; $r-0.49, P<0.05$, subcutaneous $)$ in human subjects.

It is concluded that MIC-1 is expressed in adipose tissue and secreted from adipocytes and is therefore a new adipokine. Recombinant MIC-1 enhances adiponectin release, suggesting that it is a positive regulator of adiponectin. Taken together, MIC-1 as a novel adipokine may well have a paracrine role in the modulation of adipose tissue function and body fat mass.

This work was supported by the Liverpool University R\&D Fund and the Biotechnology and Biological Sciences Research Council (grant no. BBE015379).

1. Bootcov MR, Bauskin AR, Valenzuela SM et al. (1997) Proc Natl Acad Sci U S A 94, 11514-11519.

2. Johnen H, Lin S, Kuffner T et al. (2007) Nat Med 13, 1333-1340.

3. Trayhurn P \& Bing C (2006) Philos Trans R Soc Lond B Biol Sci 361, 1237-1249. 